\section{Doucet, M. et Thériault, M. (dir.) (2019). L'adulte en formation... pour devenir soi. Espaces, passages, débats et défis. Québec, QC : Presses de I'Université du Québec.}

doi:10.18162/fp.2019.a178

\title{
RECENSION
}

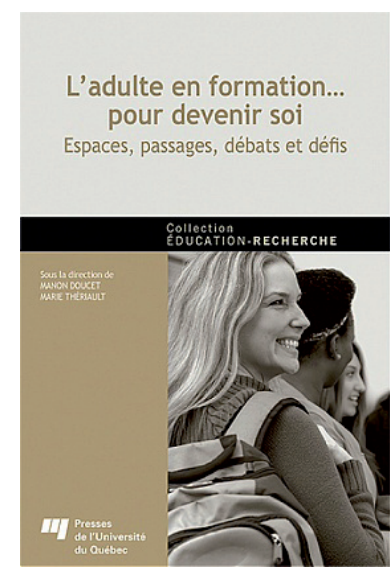

Enseigner aux adultes est un défi complexe qui exige une multitude de compétences professionnelles. Au-delà de ces compétences nécessaires, une grande capacité d'adaptation aux différents enjeux et défis s'avère plus importante que jamais, alors que la formation aux adultes, au même titre que ces adultes eux-mêmes, se transforme constamment. Sensible à cette perpétuelle mutation, le collectif dirigé par $\mathbf{M}^{\text {mes }}$ Manon Doucet et Marie Thériault, intitulé L'adulte en formation... pour devenir soi. Espaces, passages, débats et défis (2019) porte très bien son titre, alors qu'il permet au lecteur de bien saisir l'étendue des recherches effectuées en éducation depuis les 15 dernières années, en ce qui a trait à la formation aux adultes.

Ainsi, l'ouvrage organisé selon cinq axes nous plonge non seulement au cœur des espaces, passages, débats et défis, mais il le fait en nous intéressant au niveau secondaire, professionnel, collégial et universitaire, en plus d'examiner de manière significative la formation continue. Après la présentation d'un premier axe portant sur «L'éducation des adultes et les enjeux internationaux ", le second axe ayant pour thème « L'éducation des adultes et la formation générale des adultes » illustre la posture des apprenants de la formation générale des adultes, les conséquences du décrochage scolaire et les perspectives de formation générale aux adultes (chapitres 2 et 3 ). De plus, ce même axe, au niveau des chapitres 4 et 5 , nous permet de bien saisir les défis qui meublent le quotidien de ces enseignants en formation générale des adultes (FGA) ainsi que les trajectoires et les besoins en formation qui qualifient cette démarche de coconstruction réalisée avec les acteurs scolaires des centres d'éducation aux adultes (CEA). 
Au niveau du troisième axe portant sur "L'éducation des adultes, la formation professionnelle et la formation continue ", il est question des pratiques d'enseignement, du codéveloppement et des dispositifs favorisant l'insertion des enseignants (chapitres 6, 7 et 8). Ce passage sur la formation professionnelle et continue anticipe très bien le quatrième axe visant « L'éducation des adultes : le cégep et l'université ». Au sein de cet axe, deux chapitres (9 et 10) portent sur la différenciation pédagogique, l'actualisation des modèles consultatifs et sur l'élaboration des besoins rencontrés en accompagnement éducatif des étudiants en situation de handicap. Alors que le chapitre 11 illustre l'importance de la pratique réflexive comme levier de construction identitaire, le chapitre 12, lui, vient clarifier les facteurs internes et externes ayant un impact sur le retour aux études universitaires des professionnels.

Finalement, «L'éducation des adultes, la formation à l'emploi et la formation en entreprise » représente le cinquième et dernier axe de cet ouvrage qui s'avère essentiel pour couvrir ce spectre large caractérisant le parcours et les attentions particulières qui doivent être apportées en contexte d'éducation des adultes. Ce dernier axe porte d'ailleurs sur les contextes de violence vécue au niveau de la formation des adultes (chapitre 13) et ferme l'ouvrage à merveille avec une réflexion théorique sur l'homéoformation (chapitre 14). Ainsi, les enseignants qui œuvrent au sein des différents niveaux d'éducation des adultes au Québec trouveront un écho à leurs propres préoccupations, mais seront aussi sensibilisés à des enjeux relatifs à différents contextes et qui ont pu être identifiés par leurs pairs.

\section{Pour citer cet article}

Trudel, S. (2019). Doucet, M. et Thériault, M. (dir.) (2019). L'adulte en formation... pour devenir soi. Espaces, passages, débats et défis. Québec, QC : Presses de l'Université du Québec. http://dx.doi.org/10.2307/j.ctvggx4kj.

Formation et profession. 27(2), 127-128. http://dx.doi.org/10.18162/fp.2019.a178 